Open Access

\title{
Assessment of the proximity between the mandibular third molar and inferior alveolar canal using preoperative 3D-CT to prevent inferior alveolar nerve damage
}

Byeongmin Lee ${ }^{1}$, Youngju Park', Janghoon Ahn ${ }^{2^{*}}$, Jihyun Chun ${ }^{1}$, Suhyun Park', Minjin Kim¹, Youngserk Jo ${ }^{1}$, Somi Ahn', Beulha Kim and Sungbae Choi

\begin{abstract}
Background: The inferior alveolar nerve (IAN) may be injured during extraction of the mandibular third molar, causing severe postoperative complications. Many methods have been described for evaluating the relative position between the mandibular third molar and the inferior alveolar canal (IAC) on panoramic radiography and computed tomography, but conventional radiography provides limited information on the proximity of these two structures. The present study assessed the benefits of three-dimensional computed tomography (3D-CT) prior to surgical extraction of the mandibular third molar, to prevent IAN damage.
\end{abstract}

Methods: This retrospective study included 4917 extractions in 3555 patients who presented for extraction of the mandibular third molars. The cases were classified into three groups, according to anatomical relationship between the mandibular third molars and the IAC on panoramic radiography and whether 3D-CT was performed. Symptoms of IAN damage were assessed using the touch-recognition test. Data were compared using the chi-square test and Fisher's exact test.

Results: Among the 32 cases of IAN damage, 6 cases were included in group I ( $0.35 \%, n=1735$ cases), 23 cases in group II (1.1\%,n=2063 cases), and 3 cases in group III (0.27\%, n= 1119 cases). The chi-square test showed a significant difference in the incidence of IAN damage between groups I and II. No significant difference was observed between groups I and III using Fisher's exact test. In the 6 cases of IAN damage in group I, the mandibular third molar roots were located lingual relative to the IAC in 3 cases and middle relative to the IAC in 3 cases. The overlap was $\geq 2 \mathrm{~mm}$ in 3 of 6 cases and $0-2 \mathrm{~mm}$ in the remaining 3 cases. The mean distance between the mandibular third molar and IAC was $2.2 \mathrm{~mm}$, the maximum distance $12 \mathrm{~mm}$, and the minimum distance $0.5 \mathrm{~mm}$. Greater than $80 \%$ recovery was observed in 15 of $32(46.8 \%)$ cases of IAN damage.

Conclusions: 3D-CT may be a useful tool for assessing the three-dimensional anatomical relationship and proximity between the mandibular third molar and IAC in order to prevent IAN damage during extraction of mandibular third molars.

Keywords: Spiral CT; Third molar; Tooth extraction; Inferior alveolar nerve

\footnotetext{
* Correspondence: ajh0225@gmail.com

${ }^{2}$ Department of Orthodontics, Kangnam Sacred Heart Hospital, College of Medicine, Hallym University, 1, Singil-ro, Yeongdeungpo-gu, Seoul 150-950, Korea

Full list of author information is available at the end of the article
} 


\section{Background}

The inferior alveolar nerve (IAN) may be injured during extraction of the mandibular third molar, which often results in severe complications for patients [1]. The resulting neurological complications are closely correlated with the anatomical proximity of the IAN, patient age, the root curvature, and the surgical procedure employed to remove distal bone [2-4]. Therefore, many studies recommend preoperative imaging, such as panoramic radiography, standard periapical radiography, computed tomography, and scenography, before surgically removing an impacted mandibular third molar [5-13]. Many methods have been described for evaluating the relative position between the mandibular third molar and the inferior alveolar canal (IAC) on panoramic radiography and tomography [14-19]. However, conventional panoramic radiography provides only limited information on the anatomical morphology of the mandibular third molar and its anatomical relationship to the IAC.

Three-dimensional computed tomography (3D-CT) may be ideal to evaluate the close relationship between the third molars and IAC because it provides a threedimensional view. Many studies report that the proximity between the third molars and IAC is an important factor in determining the risk of IAN damage after extraction of the mandibular third molar [2, 4]. Therefore, in the present study, we evaluated the usefulness of 3D$\mathrm{CT}$ in preventing IAN damage during surgical removal of the mandibular third molars by determining its proximity to the IAC.

\section{Methods}

A total of 4917 extractions in 3555 patients were included in this retrospective study. All extractions were performed by the oral and maxillofacial surgeons at our hospital. All images were acquired using a panoramic radiographic machine (Auto III N CMR, Asahi Roentgen Ind. Co., Japan) and a 3D-CT unit (Somatom sensation 64 channel, Siemens Ind. Co., Germany). All cases in this study were classified into three groups, according to anatomical relationship between the mandibular third molars and the IAC on the panoramic view and whether a 3D-CT image were acquired (Table 1). Patients in group I exhibited an overlap between the mandibular third molar and IAC on

Table 1 Classification of cases (Y: yes, N: no)

\begin{tabular}{lll}
\hline & Overlapping $^{\text {a }}$ & 3D-CT \\
\hline Group I & Y & Y \\
Group II & Y & N \\
Group III & N & N
\end{tabular}

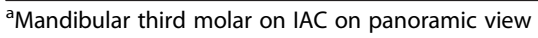

panoramic radiography and underwent spiral 3D-CT. Patients in group II also exhibited an overlap, but they did not undergo spiral 3D-CT. Patients in group III did not exhibit an overlap on panoramic radiography, so they did not undergo spiral 3D-CT. The study protocol was reviewed and approved by the Kangnam Sacred Heart Hospital Institutional Review Board (2013-12-108).

A total of 1735 cases (35.3\%) were classified into group I, 2063 cases (42\%) into group II, and 1119 cases (22.7 \%) into group III (Fig. 1). The age distributions are summarized in Figs. 2 and 3. A total 391 of 4917 cases (7.95 \%) were aged $10-19$ years; 2661 cases (54.19\%), 20-29 years; 1255 cases (25.5\%), 30-39 years; 425 cases (8.6 \%), 4049 years; 130 cases (2.6\%), 50-59 years; 38 cases $(0.77 \%)$, 60-69 years; 14 cases (0.28\%), 70-79 years; and 3 cases $(0.06 \%)$ were age $80-89$ years.

The distance between the mandibular third molar and IAC was measured using the Pi-view STAR program (INFINITT, Seoul, Korea) by a single examiner. The examiner marked a dot on each molar and the adjacent IAC, and the shortest distance between them was measured on the coronal 3D-CT image (Fig. 4).

The touch-recognition test, which measures subjective neurologic symptoms, was performed 1-2 days and 7-10 days postoperatively at the lower lip, chin, gingiva, and tongue to detect IAN or lingual nerve damage. Data were analyzed using the chi-square test and the Fisher's exact test at a threshold $p$ value of 0.05 . All statistical analyses were performed using SPSS software version 17.0 (SPSS, Chicago, IL, USA).

\section{Results}

IAN and lingual nerve damage occurred in 32 and 12 of 4917 cases, respectively (total 43 of 4917 cases; Table 2). Hypoesthesia was observed in all cases with IAN damage, but anesthesia did not occur in any case.

\section{Total 4917 cases}

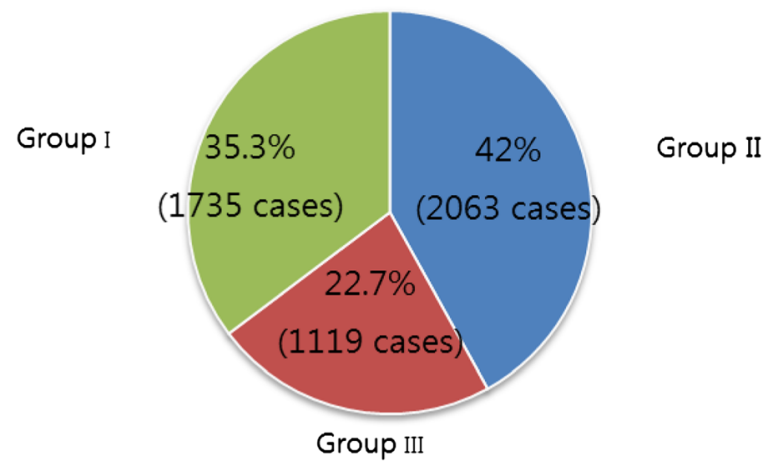

Fig. 1 Group-specific distribution of total cases 


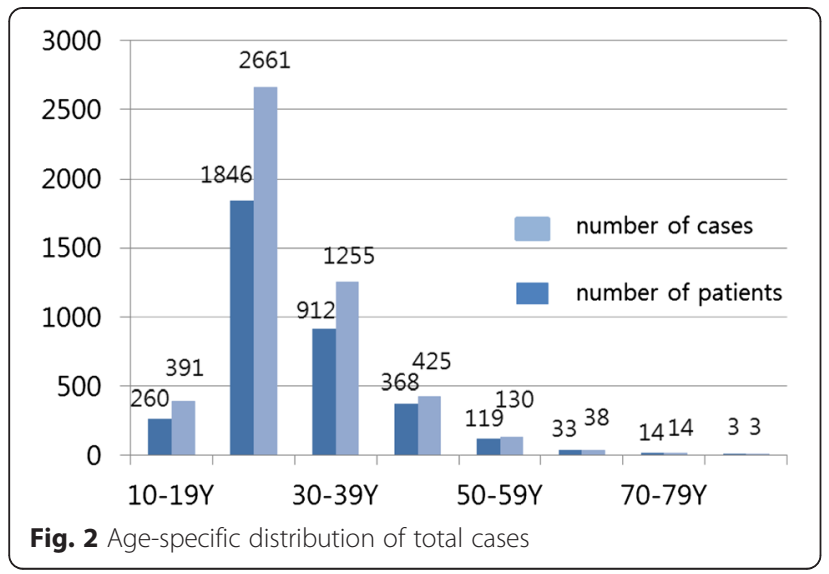

The age distribution for the cases of IAN damage was as follows: 3 cases aged $10-19$ years; 8 cases $20-$ 29 years; 12 cases $30-39$ years; 8 cases $40-49$ years; 1 case 50-59 years; and none 60 years and older (Fig. 5). Among the 32 cases of IAN damage, 21 of 32 (65.6\%) cases were right-sided, and 11 of 32 (34.4\%) were leftsided. In group I ( $n=1735$ cases), the anatomical relationship between the overlapping mandibular third molar and IAC was as follows: the mandibular third molar roots were located buccal relative to the IAC in $348(20.1 \%)$ cases; middle relative to the IAC in 437 $(25.2 \%)$ cases; and lingual relative to the IAC in 950 (54.7\%) cases (Figs. 6 and 7).

Figure 8 shows the distances between the mandibular third molar and IAC. In 96 of 1735 (5.5 \%) cases, the

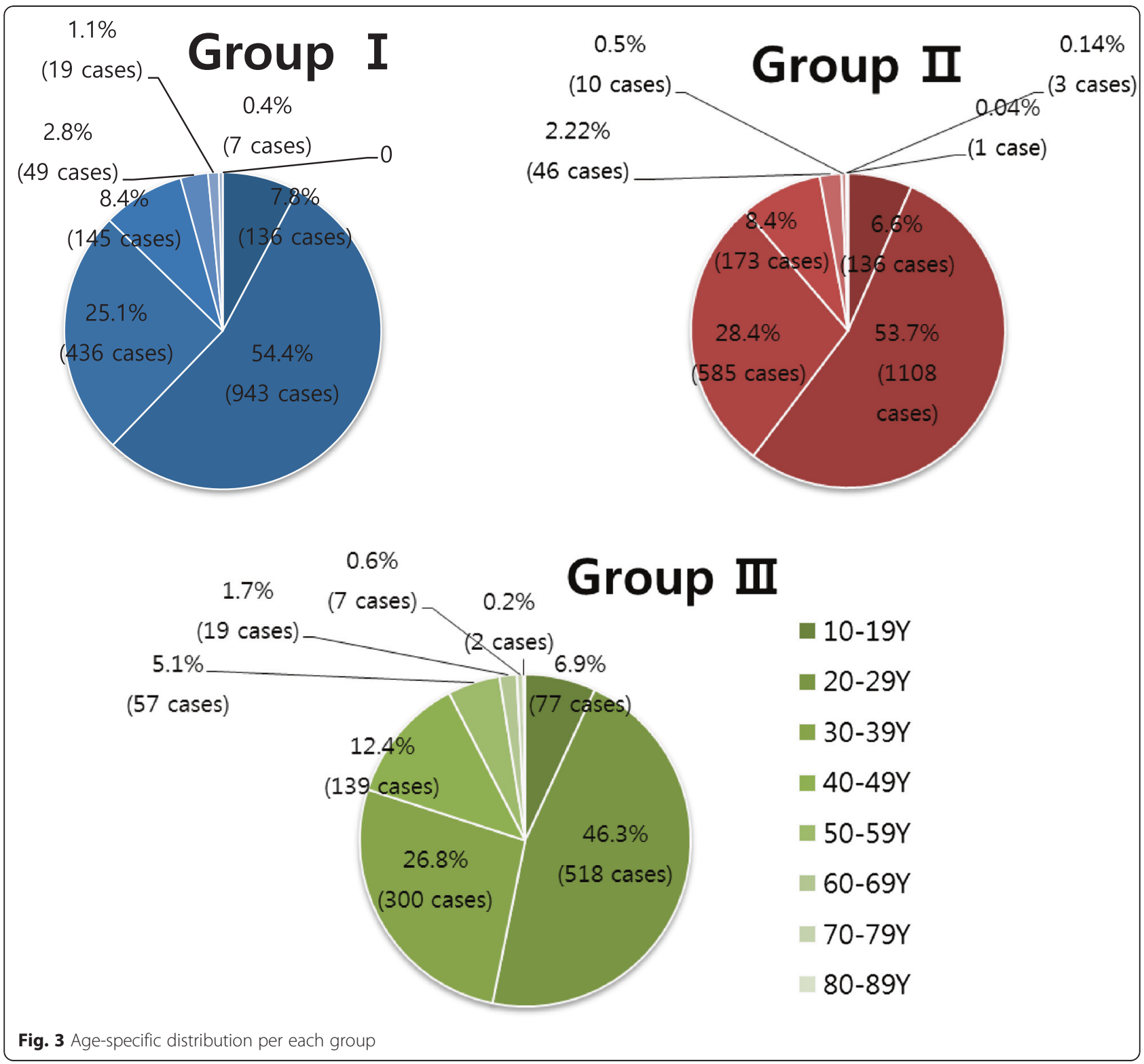




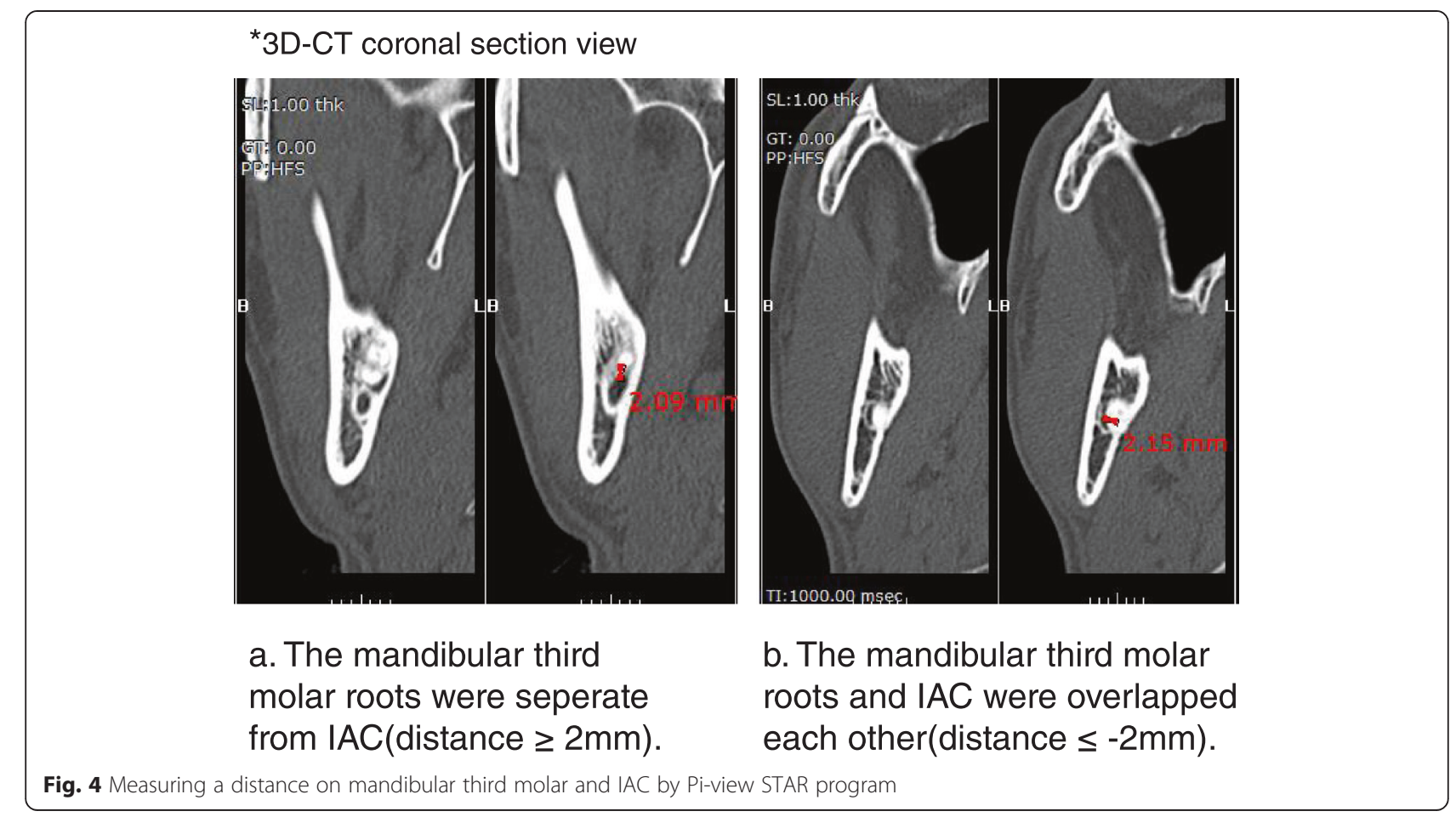

IAC and third molar root overlapped more than $2 \mathrm{~mm}$, and in 510 cases $(29.4 \%)$, they overlapped between 0 and $2 \mathrm{~mm}$. In 490 of 1735 cases $(28.3 \%)$, the IAC and mandibular third molar were between 0 and $2 \mathrm{~mm}$ apart, and in 639 cases (36.8\%), they were separated by greater than $2 \mathrm{~mm}$. In some images, the mandibular third molar roots appeared to overlap on panoramic radiography, but on $3 \mathrm{D}-\mathrm{CT}$, the IAC and third molar roots were separate from each other. In the 6 cases of IAN damage in group I, the mandibular third molar roots were located lingual relative to the IAC in 3 cases, and middle relative to the IAC in 3 cases. The distance between the mandibular third molar and IAC was also investigated in these 6 cases. Three cases showed an overlap of $2 \mathrm{~mm}$ or greater, and the remaining 3 cases showed an overlap measuring from $0 \mathrm{~mm}$ to less than $2 \mathrm{~mm}$.

In group III, the mean distance between the mandibular third molar and the IAC was $2.2 \mathrm{~mm}$. The maximum

Table 2 Incidence of lingual nerve damage and IAN damage of each group

\begin{tabular}{llll}
\hline & $\begin{array}{l}\text { Lingual nerve } \\
\text { damage }\end{array}$ & IAN damage & $\begin{array}{l}\text { IAN damage } \\
\text { rate (\%) }\end{array}$ \\
\hline Group I (1735 cases) & 3 cases & 6 cases & 0.35 \\
Group II (2063 cases) & 6 cases & 23 cases & 1.1 \\
Group III (1119 cases) & 3 cases & 3 cases & 0.27 \\
Total (4917 cases) & 12 cases & 32 cases & 0.65 \\
\hline
\end{tabular}

distance was $12 \mathrm{~mm}$, and the minimum distance was $0.5 \mathrm{~mm}$.

The recovery from IAN and lingual nerve damage was also assessed. Six of $11(54.5 \%)$ cases of lingual nerve damage recovered more than $80 \%$. Greater than $80 \%$ recovery was observed in 15 of $32(46.8 \%)$ cases of IAN damage (Table 3).

\section{Discussion}

In our study, both panoramic radiography and 3D-CT were used to evaluate the anatomic relationship between the mandibular third molar and the IAN. The mean incidence of IAN damage during extraction of

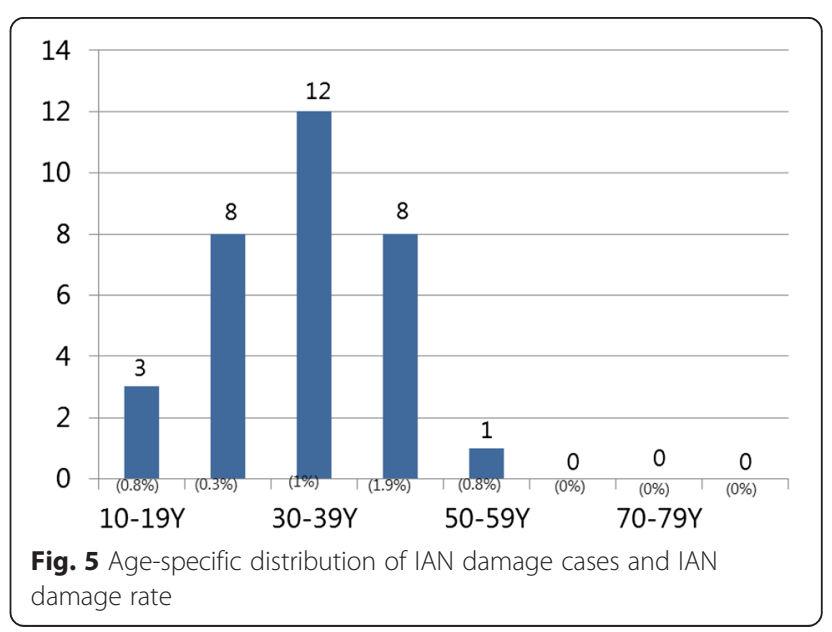




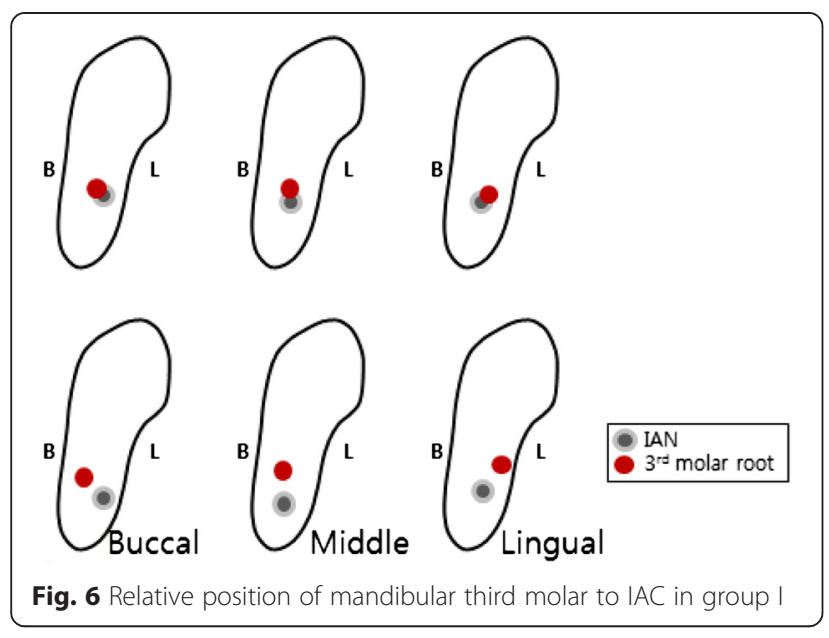

the mandibular third molar was $0.65 \%$ in our study (Table 2). The incidence of IAN damage varies among previous studies. Carmichael and McGowan [5] reported that the incidence of IAN damage was 0.4$5.5 \%$; in most cases, IAN damage recovered spontaneously, but permanent paresthesia persisted in less than $1 \%$ of cases. Bataineh [18] concluded that the incidence of IAN damage was $0.4-8.4 \%$ based on review of several previous retrospective studies.

In our study, the mandibular third molar root tended to be located lingually to the IAC (Fig. 8). Kaeppler [12] found that the IAC was located buccal relative to the mandibular third molar roots and recommended performing $\mathrm{CT}$ in addition to conventional panoramic radiography for a more accurate anatomic diagnosis. Similarly, Pawelzick et al. [13] recommended performing volumetric CT to assess the location of the mandibular third molar and IAC three-dimensionally.

In the present study, the incidence of IAN damage was $0.35 \%$ in group I, $1.1 \%$ in group II, and $0.27 \%$ in group III. Nakagawa et al. [20] asserted that it was important to know the anatomical proximity between the

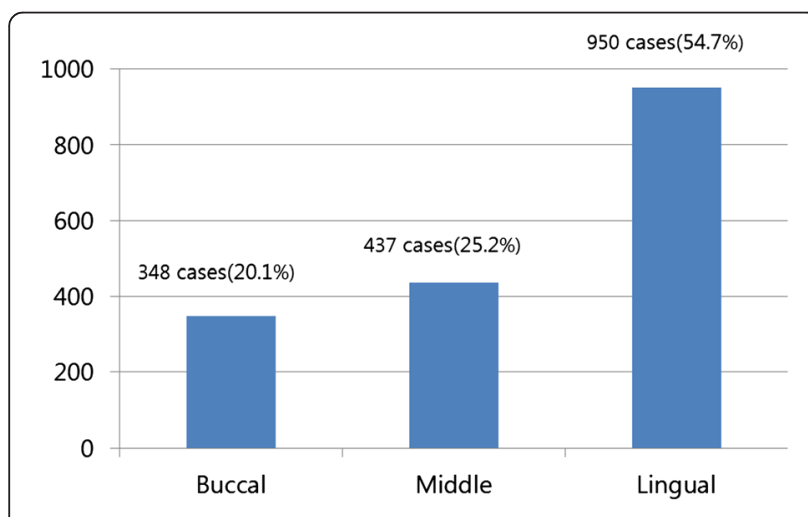

Fig. 7 Relative position of mandibular third molar to IAC in group I

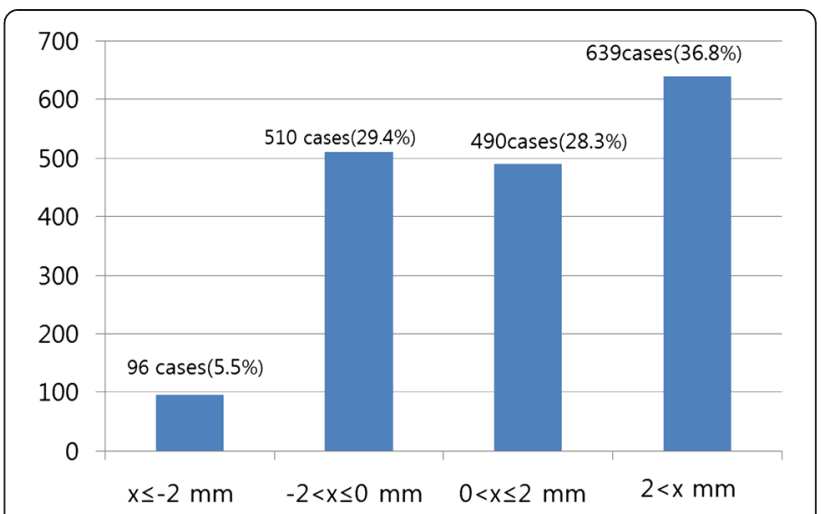

Fig. 8 The distance from mandibular third molar to IAC in group $\mid$

mandibular third molar and IAC before molar extraction as understanding this proximity was critical in preventing IAN damage.

The IAN damage rate was highest in patients aged 40-49 years. Patients aged 60 years and older were not subjected to detailed analysis in our study because none experienced IAN damage (Fig. 5). Few studies have examined patient age as a risk factor of IAN damage, though Valmaseda et al. [2] reported that the risk of IAN damage increased as patients aged. Two theories have been proposed to explain this phenomenon. Potentially, surgical trauma may be more severe in older patients, and alternatively, healing may be poorer in older patients than in younger patients. However, Kipp et al. [21] stated that there was no relationship between age and IAN damage.

In this study, the incidence of IAN damage was $0.35 \%$ in group I and $1.1 \%$ in group II and, according to the chi-square test, differed significantly between these two groups $(P<0.05)$. This result shows that using 3D-CT can reduce the incidence of IAN damage in the case of overlap between the mandibular third molar and IAC on panoramic radiography.

There was no statistically significant difference in the incidence of IAN damage between group I $(0.35 \%)$ and group III $(0.27 \%)$ according to Fisher's exact test (Table 4). This is that extraction can be performed in

Table 3 Recovery of inferior alveolar nerve damage and lingual nerve damage

\begin{tabular}{lcc}
\hline Recovery of nerve damage & Lingual nerve damage & IAN damage \\
\hline $80 \% \leq x \leq 100 \%$ & 6 cases $(54.5 \%)$ & 15 cases $(46.8 \%)$ \\
$60 \% \leq x<80 \%$ & 2 cases $(18.2 \%)$ & 3 cases $(9.4 \%)$ \\
$40 \% \leq x<60 \%$ & 0 case $(0 \%)$ & 6 cases $(18.8 \%)$ \\
$x<40 \%$ & 1 case $(9.1 \%)$ & 0 case $(0 \%)$ \\
No visit & 2 cases $(18.2 \%)$ & 8 cases $(25 \%)$ \\
Total & 11 cases $(100 \%)$ & 32 cases $(100 \%)$ \\
\hline
\end{tabular}


Table 4 Statistical analysis

\begin{tabular}{llc}
\hline & Statistical analysis & $p$ value \\
\hline Comparison of group I and group II & The chi-square test & $0.007^{*}$ \\
Comparison of group I and group III & Fisher's exact test & $1.0^{* * *}$ \\
\hline
\end{tabular}

${ }^{*}$ Statistically significant $(P<0.05)$

${ }^{* *}$ Statistically not significant $(P<0.05)$

group I with similar incidence rate of IAN damage to group III if extraction was performed after careful identification about the locations of the compared IAC and root.

Collectively, these results indicate that 3D-CT may be a useful diagnostic tool in preventing IAN damage in patients undergoing extraction of the mandibular third molar.

In addition, the removal of impacted teeth in orthognathic surgery is well-documented and recommended at least 9 to 12 months prior to the planned orthognathic surgery [22]. The findings in this study can be used to reduce IAN damage during mandibular third molar extraction before orthognathic surgery and the fracture line design using 3D-CT has to be additionally studied for orthognathic surgery with unerupted third molars.

\section{Conclusions}

Preoperative radiographic examination is necessary to determine the relationship between the mandibular third molar and IAC as it helps prevent IAN damage during extraction of the mandibular third molar. Based on the current findings, we conclude that 3DCT imaging may be a useful tool in assessing the anatomical proximity between the mandibular third molar and IAC in three-dimensions and preventing IAN damage in patients undergoing extraction of the mandibular third molar.

\section{Abbreviations}

3D-CT: three-dimensional computed tomography; IAC: inferior alveolar canal; IAN: inferior alveolar nerve.

\section{Competing interests}

The authors declare that they have no competing interests.

\footnotetext{
Authors' contributions

BL drafted the manuscript, is accountable for all aspects of the work in ensuring that questions related to the accuracy or integrity of any part of the work are appropriately investigated and resolved, and performed the statistical analysis. YP participated in the design of the study. JA participated in the design of the study and made the final approval of the version to be published. JC, SP, YJO, SA, and BK helped in the acquisition of data and drafting of the manuscript. MK helped in the acquisition of data and revised it critically for important intellectual content. SC participated in the analysis and interpretation of data, helped to draft the manuscript, and revised it critically for important intellectual content. All authors read and approved the final manuscript.
}

\section{Author details}

${ }^{1}$ Department of Oral and Maxillofacial Surgery, Kangnam Sacred Heart Hospital, College of Medicine, Hallym University, 1, Singil-ro, Yeongdeungpo-gu, Seoul 150-950, Korea. ${ }^{2}$ Department of Orthodontics, Kangnam Sacred Heart Hospital, College of Medicine, Hallym University, 1, Singil-ro, Yeongdeungpo-gu, Seoul 150-950, Korea.

Received: 26 August 2015 Accepted: 1 September 2015

Published online: 17 September 2015

\section{References}

1. Kim JH et al (2006) The study of evaluation to relationship between the inferior alveolar nerve and the mandibular third molar by using radiographic image. J Kor Oral Maxillofac Surg 32:464-473

2. Valmaseda-Castellon E, Berini-Aytes L, Gay-Escoda C (2001) Inferior alveolar nerve damage after lower third molar surgical extraction: a prospective study of 1117 surgical extractions. Oral Surg Oral Med Oral Pathol Oral Radiol Endod 92:377-383

3. Gülicher D, Gerlach KL (2001) Sensory impairment of the lingual and inferior alveolar nerves following removal of impacted mandibular third molars. Int J Oral Maxillofac Surg 30:306-312

4. Hansson PT, Fields HL, Hil RG (2001) Neuropathic pain: pathophysiology and treatment. Press in pain research and management, vol 21. IASP Press, Seattle, pp 100-172

5. Carmichael FA, McGowan DA (1992) Incidence of nerve damage following third molar removal: a West of Scotland Oral Surgery Research Group Study. Br J Oral Maxillofac Surg 30:78-82

6. Bataineh $A B$ (2001) Sensory nerve impairment following mandibular third molar surgery. J Oral Maxillofac Surg 59:1012-1017

7. Westesson PL, Carlsson LE (1980) Anatomy of mandibular third molars. A comparison between radiographic appearance and clinical observations. Oral Surg Oral Med Oral Pathol 49(1):90-4.

8. Nortjé CJ, Farman AG, Grotepass FW (1977) Variations in the normal anatomy of the inferior dental canal: a retrospective study of panoramic radiographs from 3612 routine dental patients. Br J Oral Surg 15:55-63

9. Smith AC, Barry SE, Chiong AY et al (1997) Inferior alveolar nerve damage following removal of mandibular third molar teeth: a prospective study using panoramic radiography. Aust Dent J 42(3):149-152

10. Azaz B, Shteyer A, Piamenta M (1976) Radiographic and clinical manifestations of the impacted mandibular third molar. Int J Oral Surg 5:153-160

11. Miller CS, Nummikoski PV, Barnett DA, Langlais RP (1990) Cross-section tomography. A diagnostic technique for determining the buccolingual relationship of the impacted mandibular third molars and the inferior alveolar neurovascular bundle. Oral Surg Oral Med Oral Pathol 70:791-797

12. Kaeppler $\mathrm{G}$ (2000) Conventional cross-sectional tomographic evaluation of mandibular third molars. Quintessence Int 31(1):49-56

13. Pawelzik J, Cohnen M, Willers R, Becker J (2002) A comparison of conventional panoramic radiographs with volumetric computed tomography images in the preoperative assessment of impacted mandibular third molars. J Oral Maxillofac Surg 60:979-984

14. Howe GL, Poyton HG (1960) Prevention of damage to the inferior dental nerve during the extraction of mandibular third molars. Br Dent J 109:355-363

15. Rud J (1970) The split bone technic for removal of impacted mandibular third molars. J Oral Surg 28:416-421

16. Sedaghatfar M, August MA, Dodson TB (2005) Panoramic radiographic findings as predictors of inferior alveolar nerve exposure following third molar extraction. J Oral Maxillofac Surg 63:3-7

17. Maegawa H, Sano K, Kitagawa Y et al (2003) Preoperative assessment of the relationship between the mandibular third molar and the mandibular canal by axial computed tomography with coronal and sagittal reconstruction. Oral Surg Oral Med Oral Pathol Oral Radiol Endod 96:639-646

18. Mahasantipiya PM, Savage NW, Monsour PAJ, Wilson RJ (2005) Narrowing of the inferior dental canal in relation to the lower third molars. Dentomaxillofac Radiol 34(3):154-163

19. Chye EP, Young IG, Osborne GA, Rudkin GE (1993) Outcomes after same-day oral surgery: a review of 1,180 cases at a major teaching hospital. J Oral Maxillofac Surg 51:846-849

20. Nakagawa $Y$ (2007) Third molar position: reliability of panoramic radiography. J Oral Maxillofac Surg 65:1303-1308 
21. Kipp DP, Goldstein BH, Weiss WW Jr (1980) Dysesthesia after mandibular third molar surgery: a retrospective study and analysis of 1,377 surgical procedures. J Am Dent Assoc 100:185-192

22. Johan PR, Peter $T$ (2002) Age as a factor in the complication rate after removal of unerupted/impacted third molars at the time of mandibular sagittal split osteotomy. J Oral Maxillofac Surg 60:654-659

Submit your manuscript to a SpringerOpen ${ }^{\odot}$ journal and benefit from:

- Convenient online submission

- Rigorous peer review

- Immediate publication on acceptance

- Open access: articles freely available online

- High visibility within the field

- Retaining the copyright to your article

Submit your next manuscript at $\mathbf{s p r i n g e r o p e n . c o m ~}$ 